\title{
THE DYNAMICS OF ELLIPTICAL RINGS
}

\author{
NiCOLE BORDERIES \\ Division of Geological and Planetary Sciences, California Institute of Technology, Pasadena, California 91125 and NASA/Ames Research \\ Center, Moffett Field, California 94035
}

Peter Goldreich

Division of Geological and Planetary Sciences, California Institute of Technology, Pasadena, California 91125

SCOTT TREMAINE ${ }^{\text {a) }}$

Department of Physics, Center for Space Research and Center for Theoretical Physics, Massachusetts Institute of Technology, Cambridge, Massachusetts 02139

Received 24 May 1983

\begin{abstract}
We investigate the evolution of eccentric rings under the influence of (1) differential precession due to the planetary quadrupole moment; (2) self-gravity; (3) viscous forces due to interparticle collisions; and (4) eccentricity excitation by shepherd satellites. The principal conclusions are that: (a) Uniform precession can be enforced by self-gravity (Goldreich and Tremaine 1979b); the resulting configuration is both dynamically and secularly stable. (b) Due to viscous forces the line of apsides at the inner ring edge is not exactly aligned with the line of apsides at the outer edge; the apse shift may be detectable in the $\alpha$ and $\beta$ rings of Uranus. (c) The mean eccentricity is determined by a balance between viscous damping and excitation by shepherds. (d) We expect the dimensionless eccentricity gradient $a \Delta e / \Delta a$ to be positive and of order unity in most eccentric rings, as observed.
\end{abstract}

\section{INTRODUCTION}

Several of the rings in both the Saturn and Uranus ring systems are known to be elliptical. The inner and outer boundaries of these rings have been fit by aligned Keplerian ellipses with a common line of apsides $\widetilde{\omega}$ and with eccentricities and semimajor axes $e \pm \Delta e$ and $a \pm \Delta a$; the parameters $e, a, 2 \Delta e$, and $2 \Delta a$ are given for several elliptical rings in Table I (data from Nicholson $e t$ al. 1982; Porco et al. 1983; Esposito et al. 1983; Porco 1983).

A number of different dynamical effects governs the behavior of elliptical rings. (1) Differential precession due to the planetary quadrupole moment tends to destroy the observed apse alignment. (2) Apse alignment is probably maintained by the self-gravity of the ring (Goldreich and Tremaine 1979b; hereafter Paper I). (3) Nearby "shepherd" satellites can pump up the ring eccentricity (Goldreich and Tremaine 1981). (4) Viscous forces caused by interparticle collisions damp the ring eccentricity.

In this paper we derive a set of dynamical equations for narrow elliptical rings which incorporate these effects (Sec. II). Our aim is to model the origin of the eccentricity and the evolution of elliptical rings. In Sec. III we describe a simple analytic "two-streamline" model which displays most of the important physical effects of more accurate numerical models. In Sec. IV we briefly describe " $N$-streamline" models, although detailed numerical results are reserved for a future paper. Section V

\footnotetext{
a) Alfred P. Sloan Research Fellow.

1560 Astron. J. 88 (10), October 1983
}

contains a discussion.

In our equations we consider only changes in the eccentricities and apses $e_{i}$ and $\widetilde{\omega}_{i}$ of the ring particles. We assume that our rings have zero inclination, although many elliptical rings are also inclined (French et al. 1982) and many of our equations can be applied to inclined rings with only minor changes (Borderies et al. 1983a). A more important caveat is that we do not consider changes in the semimajor axes $a_{i}$ of the ring particles. Viscous forces lead to radial spreading of an unconfined ring; shepherd satellites oppose this effect (Goldreich and Tremaine 1979a). We assume that the ring is an equilibrium so that $d a_{i} / d t=0$.

\section{EQUATIONS OF MOTION}

We work in cylindrical coordinates $(r, \phi, z)$ with the ring in the $z=0$ plane. The ring is considered to be a collection of $N$ streamlines, each specified by its mass $m_{i}$, semimajor axis $a_{i}$, eccentricity $e_{i}$, and azimuth of periapse, $\widetilde{\omega}_{i}, i=1, \ldots, N$. The position of a ring particle is specified by its true anomaly $f$ or by the azimuth $\phi=f+\widetilde{\omega}_{i}$. We assume $e_{i} \ll 1$ so that the shape of each streamline may be written as

$$
r_{i}=a_{i}\left(1-e_{i} \cos f\right) .
$$

We use the symbol $\Delta_{i j}$ to denote differences between streamlines $i$ and $j$; thus $\Delta a_{i j}=a_{i}-a_{j}$, etc. The radial separation between streamlines at azimuth $\phi$ is

where

$$
\Delta r_{i j}=J_{i j} \Delta a_{i j} \text {, }
$$

$$
\begin{gathered}
J_{i j}=1-q_{i j} \cos \left(\phi-\widetilde{\omega}_{i}+\gamma_{i j}\right), \\
0004-6256 / 83 / 101560-09 \$ 00.90 \\
\text { (c) } 1983 \text { Am. Astron. Soc. }
\end{gathered}
$$


TABLE I. Parameters of eccentric rings.

\begin{tabular}{|c|c|c|c|c|c|}
\hline & \multirow{2}{*}{\multicolumn{3}{|c|}{ Uranus }} & \multicolumn{2}{|c|}{ Saturn } \\
\hline & & & & $\begin{array}{l}\text { Titan } \\
\text { ringlet }\end{array}$ & $\begin{array}{l}\text { Huygens } \\
\text { ringlet }\end{array}$ \\
\hline $\begin{array}{l}a(\mathrm{~km}) \\
2 \Delta a(\mathrm{~km}) \\
e \\
2 \Delta e\end{array}$ & $\begin{array}{l}44751 \\
7.1 \\
6.65 \times 10^{-4} \\
4.3 \times 10^{-5}\end{array}$ & $\begin{array}{l}45694 \\
8.1 \\
4.5 \times 10^{-4} \\
7.9 \times 10^{-5}\end{array}$ & $\begin{array}{l}51180 \\
59.3 \\
7.94 \times 10^{-3} \\
7.5 \times 10^{-4}\end{array}$ & $\begin{array}{l}77871 \\
25.0 \\
2.6 \times 10^{-4} \\
1.4 \times 10^{-4}\end{array}$ & $\begin{array}{l}87491 \\
64.0 \\
3.4 \times 10^{-4} \\
3.4 \times 10^{-4}\end{array}$ \\
\hline$\frac{a \Delta e}{\Delta a}$ & 0.27 & 0.45 & 0.65 & 0.44 & 0.46 \\
\hline $\begin{array}{l}\Delta a \\
m(\mathrm{~g}) \\
2 \pi / \Omega(\mathrm{yr}) \\
\lambda_{1}^{-1}(\mathrm{yr})^{\mathrm{a}} \\
2 \Delta \tilde{\omega}^{\mathrm{a}} \\
\gamma^{\mathrm{a}}\end{array}$ & $\begin{array}{l}4.8 \times 10^{16} \\
53 \\
93 \\
5^{\circ}\end{array}$ & $\begin{array}{c}2.1 \times 10^{16} \\
139 \\
100 \\
-2^{\circ} .2 \\
13^{\circ} \\
\text { (Nicholson et al. } 1982 \text { ) }\end{array}$ & $\begin{array}{l}7.0 \times 10^{18} \\
17 \\
3.3 \times 10^{3} \\
-0.004 \\
0.05\end{array}$ & $\begin{array}{l}2.5 \times 10^{18} \\
23 \\
1.1 \times 10^{3} \\
-0.10 \\
0^{\circ} .19 \\
(\text { Porco et } \\
\text { al. } 1983 ; \\
\text { Porco 1983) }\end{array}$ & $\begin{array}{l}1.2 \times 10^{19} \\
28 \\
5.9 \times 10^{3} \\
-0.04 \\
0.04 \\
\text { (Esposito } \\
\text { et al. } 1983 ; \\
\text { Porco 1983) }\end{array}$ \\
\hline
\end{tabular}

$\overline{\bar{a}}\left|\lambda_{1}^{-1}\right|, \Delta \tilde{\omega}$, and $\gamma$ evaluated for a velocity dispersion of $v=0.1 \mathrm{~cm} / \mathrm{s} ; \lambda_{1}, \Delta \tilde{\omega}$ and $\tan \gamma$ are proportional to $v^{2}$ if $\left|\lambda_{1}\right|,\left|\lambda_{2}\right| \ll \Omega$ (see text).

$$
\begin{aligned}
q_{i j} \cos \gamma_{i j}= & \frac{a_{i} e_{i}-a_{j} e_{j} \cos \Delta \widetilde{\omega}_{i j}}{\Delta a_{i j}}, \\
q_{i j} \sin \gamma_{i j}= & -\frac{\mathrm{a}_{j} e_{j} \sin \Delta \widetilde{\omega}_{i j}}{\Delta \mathrm{a}_{i j}}, \\
& -\frac{\pi}{2} \leqslant \gamma_{i j} \leqslant \frac{\pi}{2} .
\end{aligned}
$$

Note that $\gamma_{i j} \neq \gamma_{j i}$, but $\left|q_{i j}\right|=\left|q_{j i}\right|$. In fact $q_{i j}=-q_{j i}$ if $\cos \Delta \widetilde{\omega}_{i j}<\min \left(a_{i} e_{i}, a_{j} e_{j}\right) / \max \left(a_{i} e_{i}, a_{j} e_{j}\right) ;$ otherwise $q_{i j}=q_{j i}$.

The analogous definitions in the continuum limit are

$$
\begin{aligned}
& \frac{\partial r}{\partial a}(a, \phi)=J(a, \phi), \\
& J(a, \phi)=1-q(a) \cos [\phi-\widetilde{\omega}(a)+\gamma(a)], \\
& q \cos \gamma=\frac{d(a e)}{d a}, q \sin \gamma=-a e \frac{d \widetilde{\omega}}{d a} .
\end{aligned}
$$

We assume that the ring is narrow, $\left|\Delta a_{i j}\right| \ll a_{i}$. Thus, for many purposes we can replace $a_{i}$ by the mean semimajor axis of the ring, denoted by $a$. The mean angular speed is $n=\left(G M / a^{3}\right)^{1 / 2}$, where $M$ is the planet mass.

\section{a) Self-Gravity}

As shown in Borderies et al. (1983b), the gravitational force from streamline $j$ causes secular changes in $e_{i}$ and $\widetilde{\omega}_{i}$ given by

$$
\begin{aligned}
& \left(\frac{d e_{i}}{d t}\right)_{s g}=-C_{i j} H\left(q_{i j}^{2}\right) e_{j} \sin \Delta \widetilde{\omega}_{i j}, \\
& \left(\frac{d \widetilde{\omega}_{i}}{d t}\right)_{s g}=+C_{i j} H\left(q_{i j}^{2}\right)\left(1-\frac{e_{j}}{e_{i}} \cos \Delta \widetilde{\omega}_{i j}\right),
\end{aligned}
$$

where

$$
C_{i j}=\frac{m_{j}}{\pi M}\left(\frac{a}{\Delta a_{i j}}\right)^{2} n,
$$

$$
H\left(q^{2}\right)=\frac{1-\left(1-q^{2}\right)^{1 / 2}}{q^{2}\left(1-q^{2}\right)^{1 / 2}},
$$

and we have assumed that $\left|q_{i j}\right|<1$, which is the condition that the streamlines do not cross.

\section{b) Quadrupole Moment of Planet}

The quadrupole moment of the planet causes apse precession at a rate

$$
\left(\frac{d \widetilde{\omega}_{i}}{d t}\right)_{p}=\frac{3}{2} J_{2} R^{2}(G M)^{1 / 2} a_{i}^{-7 / 2},
$$

where $J_{2}$ and $R$ are the dynamical oblateness and the planetary radius. Since the ring is narrow, we may write to sufficient accuracy

$$
\left(\frac{d \widetilde{\omega}_{i}}{d t}\right)_{p}=-\frac{21}{4} J_{2}\left(\frac{R}{a}\right)^{2} n \frac{\Delta a_{i 1}}{a}+K,
$$

where $K$ is a constant. The quadrupole moment has no effect on the eccentricity:

$$
\left(\frac{d e_{i}}{d t}\right)_{p}=0
$$

\section{c) Viscosity}

The viscous forces within the ring are described by the pressure tensor $p_{\alpha \beta}(r, \phi, z)$. For our purposes we need only the vertically integrated pressure tensor

$$
P_{\alpha \beta}(r, \phi)=\int_{-\infty}^{\infty} d z p_{\alpha \beta}(r, \phi, z) .
$$

The force per unit length exerted on a streamline by the material outside it is $-\hat{n} \cdot \hat{e}_{a} P_{\alpha \beta} \hat{e}_{\beta}$, where repeated indices are summed from 1 to 3 . The unit vector $\hat{n}$ points outward in the ring plane, normal to the streamline, and $\left\{\hat{e}_{a}\right\}=\{\hat{r}, \hat{\phi}, \hat{z}\}$. Since $e \ll 1, \hat{n} \simeq \hat{r}$, and the mass per unit length in streamline $i$ is approximately $m_{i} / 2 \pi a$. Hence the force per unit mass on streamline $i$ from material outside it is

$$
\mathbf{F}=S \hat{r}+T \hat{\phi},
$$


where

$$
S=-\frac{2 \pi a}{m_{i}} P_{r r}, T=-\frac{2 \pi a}{m_{i}} P_{r \phi}
$$

The details of evaluating $P_{r r}$ and $P_{r \phi}$, as well as the contribution from material inside the streamline, will be discussed below. The rates of change of the orbital elements are given by Gauss's equations

$$
\begin{aligned}
& \frac{d e}{d t}=\frac{1}{n a}[S \sin f+2 T \cos f+O(e)], \\
& \frac{d \tilde{\omega}}{d t}=\frac{1}{n a e}[-S \cos f+2 T \sin f+O(e)] .
\end{aligned}
$$

Substituting from Eq. (11), dropping the terms which are $O(e)$, and averaging over one orbit,

$$
\begin{aligned}
& \left(\frac{d e_{i}}{d t}\right)_{\text {visc }}=-\frac{2 \pi}{n m_{i}}\left(\left\langle P_{r r} \sin f\right\rangle+2\left\langle P_{r \phi} \cos f\right\rangle\right), \\
& \left(\frac{d \widetilde{\omega}_{i}}{d t}\right)_{\text {visc }}=\frac{2 \pi}{n m_{i} e_{i}}\left(\left\langle P_{r r} \cos f\right\rangle-2\left\langle P_{r \phi} \sin f\right\rangle\right),
\end{aligned}
$$

where

$$
\langle X\rangle=(2 \pi)^{-1} \int_{0}^{2 \pi} X d f .
$$

The components of the pressure tensor are evaluated by solving the viscous-stress equations (Borderies et al. 1983c). The most important assumptions are the following: (1) the ring particles are identical spheres of radius $s$ and density $\rho ;(2)$ collisions conserve relative tangential velocity but reduce the absolute value of the relative normal velocity by the coefficient of restitution $\epsilon$, which is assumed to be constant along a streamline; (3) the distribution function is a triaxial Gaussian in velocity space and the velocity ellipsoid is independent of $z ;(4)$ the eccentricity $e \ll 1$, although $|q|$ may be of order unity. The solution for the pressure tensor may be written in the form

$$
\boldsymbol{P}_{\alpha \beta}=\langle\Sigma\rangle v^{2} Q_{\alpha \beta}(q,\langle\tau\rangle, f+\gamma),
$$

where $q$ and $\gamma$ are defined in Eq. (4), and $\langle\tau\rangle$ and $\langle\Sigma\rangle$ are the azimuthally averaged optical depth and surface density. The coefficient of restitution $\epsilon$ is a function of $q$ and $\langle\tau\rangle$. The mean-square one-dimensional velocity dispersion $v^{2}$ is a free parameter*; for consistency $Q_{\alpha \beta}$ must satisfy the normalization condition

$$
\left\langle Q_{r r}+Q_{\phi \phi}+Q_{z z}\right\rangle=3
$$

The pressure tensor affects the orbital elements $e_{i}$ and $\widetilde{\omega}_{i}$ only through the moments

$$
\left\langle P_{\alpha \beta} \sin f\right\rangle \equiv\langle\Sigma\rangle v^{2}\left(\mathscr{S}_{\alpha \beta} \cos \gamma-\mathscr{C}_{\alpha \beta} \sin \gamma\right),
$$

\footnotetext{
*In fact the velocity dispersion is determined indirectly through the dependence of the coefficient of restitution on impact velocity (Goldreich and Tremaine 1978).
}

$$
\left\langle P_{\alpha \beta} \cos f\right\rangle \equiv\langle\Sigma\rangle v^{2}\left(\mathscr{C}_{\alpha \beta} \cos \gamma+\mathscr{S}_{\alpha \beta} \sin \gamma\right),
$$

where

$$
\begin{aligned}
& \mathscr{S}_{\alpha \beta}(q,\langle\tau\rangle)=\left\langle\sin (f+\gamma) Q_{\alpha \beta}(q,\langle\tau\rangle, f+\gamma)\right\rangle, \\
& \mathscr{C}_{\alpha \beta}(q,\langle\tau\rangle)=\left\langle\cos (f+\gamma) Q_{\alpha \beta}(q,\langle\tau\rangle, f+\gamma)\right\rangle .
\end{aligned}
$$

In Fig. 1 we plot $\mathscr{S}_{\alpha \beta}, \mathscr{C}_{\alpha \beta}$ as a function of $q>0$ for $\langle\tau\rangle=0.1,0.5$, and 1.0. Note that $\mathscr{S}_{\alpha \beta}$ and $\mathscr{C}_{\alpha \beta}$ are odd functions of $q$.

To apply Eqs. (13) and (16) we must evaluate $\mathscr{S}_{\alpha \beta}$ and $\mathscr{C}_{\alpha \beta}$ at the outer edge of streamline $i$. To do so we set $q=q_{i, i+1}$ and $\gamma=\gamma_{i, i+1}$. To evaluate $\langle\Sigma\rangle$ and $\langle\tau\rangle$ we assign a mass $\frac{1}{2}\left(m_{i}+m_{i+1}\right)$ to the annulus enclosed by streamlines $i$ and $i+1$; then from Eqs. (2) and (3)

$$
\begin{aligned}
\langle\Sigma\rangle & =\frac{m_{i}+m_{i+1}}{4 \pi a}\left\langle\frac{1}{\left|\Delta r_{i, i+1}\right|}\right\rangle \\
& =\frac{m_{i}+m_{i+1}}{4 \pi a\left|\Delta a_{i, i+1}\right|}\left(1-q_{i, i+1}^{2}\right)^{-1 / 2},
\end{aligned}
$$

and

$$
\langle\tau\rangle=\frac{3}{4 \rho s}\langle\Sigma\rangle .
$$

Finally, an analogous procedure is used to determine the changes in orbital elements of streamline $i$ due to the ring material inside it.

\section{d) Shepherd Satellites}

For simplicity we consider a satellite on a circular orbit. The satellite has mass $m_{s}$ and semimajor axis $a_{s}$, and we assume that $\left|a_{i}-a_{s}\right| \ll a$ and $a e_{i} \ll\left|a_{i}-a_{s}\right|$ (for more general formulae see Goldreich and Tremaine 1981).

The satellite causes secular changes in the eccentricity of streamline $i$ at a rate

$$
\frac{d e_{i}}{d t}=f\left(\frac{m_{s}}{M}\right)^{2} n\left|\frac{a}{a_{i}-a_{s}}\right|^{5} e_{i} .
$$

The coefficient $f$ is rather uncertain. In the limit where $m_{s}$ is sufficiently small so that all resonant perturbations in the ring are linear, $f=-0.074$. If the dominant corotation resonances saturate, $f=1.523$. If gaps open at the dominant Lindblad resonances, $f=0.761$. We shall assume that $f>0$ so that the eccentricity grows, but we are not concerned with the exact value of $f$.

The contribution of the satellite to $d \widetilde{\omega} / d t$ is probably negligible compared to the contribution of the planet's quadrupole moment (cf. Paper I).

\section{A SIMPLE MODEL SYSTEM}

To clarify the physics we consider a simple idealized ring in which small quantities can be ordered by two parameters $\epsilon_{1}$ and $\epsilon_{2}$ satisfying $\epsilon_{1} \ll \epsilon_{2} \ll 1$. Our model ring is based on the following approximations: (1) There are only two streamlines, with equal masses $m_{1}=m_{2}=\frac{1}{2} m$. We write $\Delta a_{21} \equiv \Delta a>0$, where 


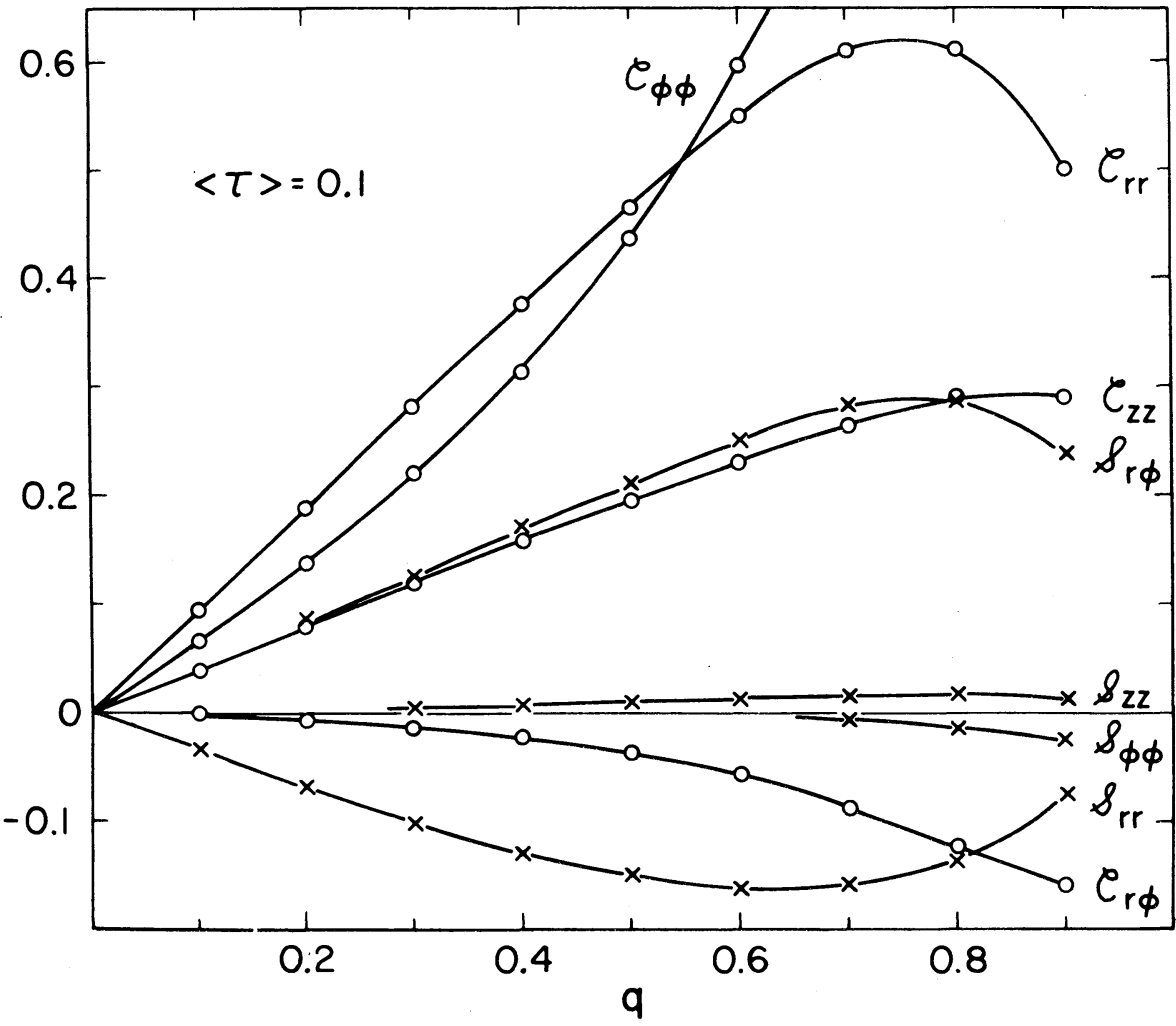

FIG. 1(a). Plots of the parameters $\mathscr{C}_{\alpha \beta}$ and $\mathscr{S}_{\alpha \beta}$ defined in Eqs. (17) and (18) for azimuthally averaged optical depth $\langle\tau\rangle=0.1$.

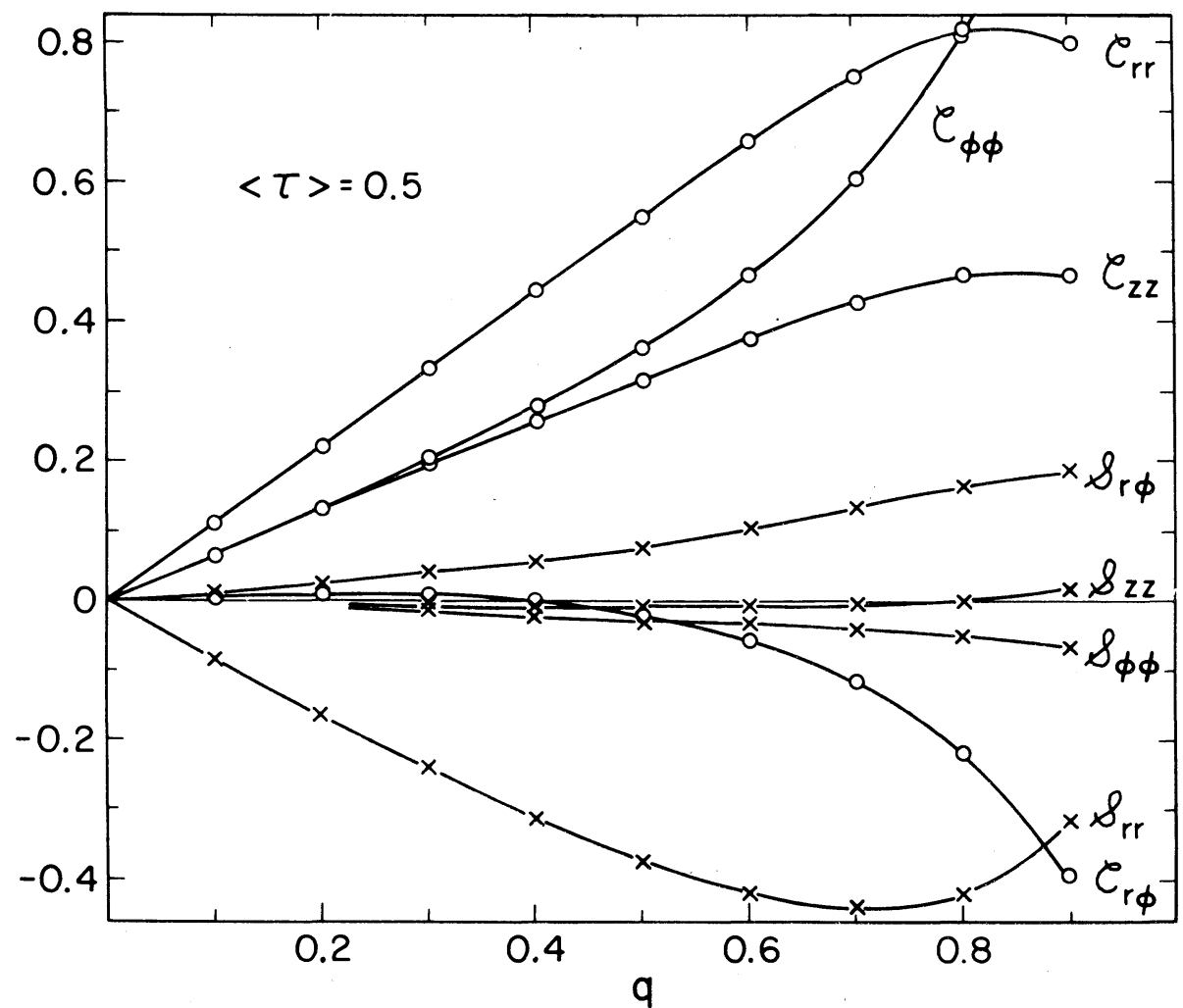

Fig. 1(b). Plots of the parameters $\mathscr{C}_{\alpha \beta}$ and $\mathscr{S}_{\alpha \beta}$ defined in Eqs. (17) and (18) for azimuthally averaged optical depth $\langle\tau\rangle=0.5$. 


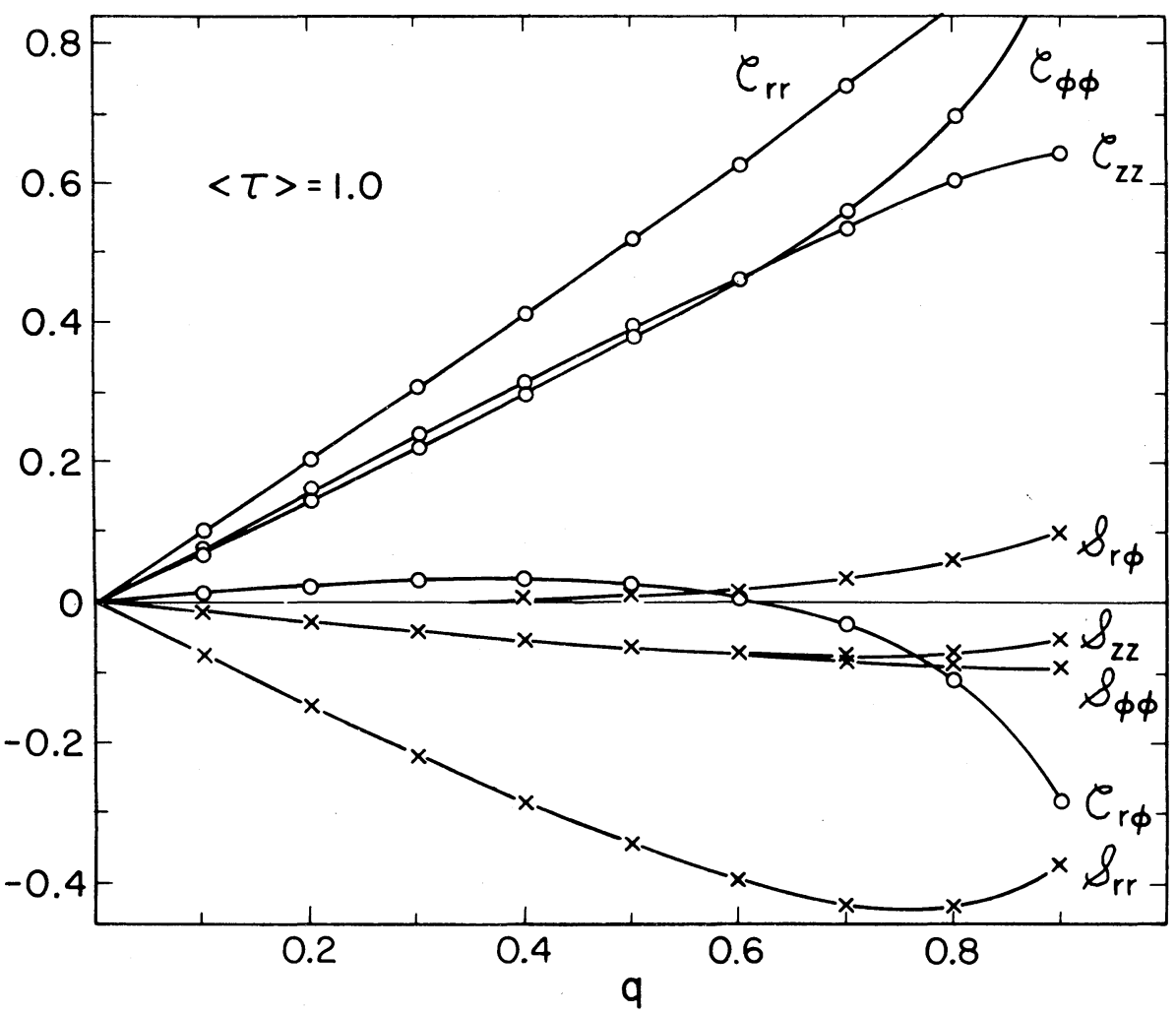

FIG. 1(c). Plots of the parameters $\mathscr{C}_{\alpha \beta}$ and $\mathscr{S}_{\alpha \beta}$ defined in Eqs. (17) and (18) for azimuthally averaged optical depth $\langle\tau\rangle=1.0$.

$\Delta a / a=O\left(\epsilon_{1}\right) \cdot(2)$ The mean eccentricity $e=\frac{1}{2}\left(e_{1}+e_{2}\right)$ is small, $e=\boldsymbol{O}\left(\epsilon_{2}\right)$. Furthermore the eccentricity difference $\Delta e_{21} \equiv \Delta e$ is assumed to be $O\left(\epsilon_{1}\right)$. Thus $\Delta e /$ $e=O\left(\epsilon_{1} / \epsilon_{2}\right) \ll 1$. (3) The difference in periapse between the streamlines is small. We assume that $\Delta \widetilde{\omega}_{21} \equiv \Delta \widetilde{\omega}=O(\Delta e / e)=O\left(\epsilon_{1} / \epsilon_{2}\right) \ll 1$. In the course of the calculations below we shall see that this ordering is self-consistent. For comparison with observations we identify $\Delta e$ and $\Delta a$ with half of the variation in $e$ and $a$ across a ring (see Table I). Note that the approximations $\Delta a / a \ll 1$ and $e \ll 1$ are all very well satisfied by the rings with measured $e$ and $\Delta e$ in the Saturnian and Uranian systems; $\Delta e / e$ ranges from 0.03 to 0.5 so the approximation $\Delta e / e<1$ is not too bad.

With this ordering $q_{12}=q_{21}(\equiv q)$, and we have

$$
q^{2}=\left(\frac{a \Delta e}{\Delta a}\right)^{2}+\left(\frac{a e \Delta \widetilde{\omega}}{\Delta a}\right)^{2}+O\left(\epsilon_{2}, \frac{\epsilon_{1}^{2}}{\epsilon_{2}^{2}}\right) \text {. }
$$

Note that $O(q)=1$. The difference

$$
\gamma_{12}-\gamma_{21} \simeq-\Delta \widetilde{\omega}=O\left(\frac{\epsilon_{1}}{\epsilon_{2}}\right)
$$

hence we may set $\gamma_{12} \simeq \gamma_{21} \equiv \gamma$, where

$$
\tan \gamma=-\frac{e \Delta \widetilde{\omega}}{\Delta e}+O\left(\epsilon_{2}, \frac{\epsilon_{1}}{\epsilon_{2}}\right)
$$

We shall also use the relations

$$
q \cos \gamma=\frac{a \Delta e}{\Delta a}+O\left(\epsilon_{2}, \frac{\epsilon_{1}}{\epsilon_{2}}\right)
$$

$$
q \sin \gamma=-\frac{a e \Delta \widetilde{\omega}}{\Delta a}+O\left(\frac{\epsilon_{1}}{\epsilon_{2}}\right)
$$

\section{a) Libration}

First we neglect viscous forces and the influence of shepherds, and keep only the lowest order nonvanishing terms in $\Delta e$ and $\Delta \widetilde{\omega}$. The equations of motion for $e_{1}, e_{2}$, and $\Delta \widetilde{\omega}$ (Eqs. 5 and 8 ) become

$$
\begin{aligned}
\left(\frac{d e_{1}}{d t}\right)_{s g+p} \simeq & -\left(\frac{d e_{2}}{d t}\right)_{s g+p} \\
\simeq & \frac{m}{2 \pi M}\left(\frac{a}{\Delta a}\right)^{2} n H\left(q^{2}\right) e \Delta \widetilde{\omega}, \\
\left(\frac{d \Delta \widetilde{\omega}}{d t}\right)_{s g+p} \simeq & \frac{m}{\pi M}\left(\frac{a}{\Delta a}\right)^{2} n H\left(q^{2}\right) \frac{\Delta e}{e} \\
& -\frac{21}{4} J_{2}\left(\frac{R}{a}\right)^{2} n \frac{\Delta a}{a} .
\end{aligned}
$$

Thus $e=\frac{1}{2}\left(e_{1}+e_{2}\right)$ satisfies $(d e / d t)_{s g+p}=0$ to lowest order in $\Delta e$ and $\Delta \widetilde{\omega}$ and we may take $e=$ constant (but see Eq. 37 for a more accurate expression). We have

$$
\left(\frac{d \Delta e}{d t}\right)_{s g+p} \simeq-\frac{m}{\pi M}\left(\frac{a}{\Delta a}\right)^{2} n H\left(q^{2}\right) e \Delta \widetilde{\omega} .
$$

The equilibrium condition $(d \Delta \widetilde{\omega} / d t)_{s g+p}$ $=(d \Delta e / d t)_{s g+p}=0$ yields

$$
\Delta \widetilde{\omega}_{s g+p}^{0}=0 \text {, }
$$




$$
\Delta e_{s g+p}^{0}=\frac{21 \pi e}{4} J_{2} \frac{M}{m}\left(\frac{R}{a}\right)^{2}\left(\frac{\Delta a}{a}\right)^{3} \frac{1}{H\left(q^{2}\right)} .
$$

Note that $\Delta e^{0}>0$, as is observed in all of the rings in Table I. A more accurate version of Eq. (28), for a ring with $N$ streamlines, was used to estimate the mass of the $\epsilon$ ring in Paper I. We give ring masses obtained from Eq. (28) in Table I; the results of $N$-streamline calculations suggest that these masses may be too large by about a factor of 2 .

The general solution of Eqs. (26) and (27) for small oscillations around equilibrium is

$$
\begin{aligned}
& \Delta \tilde{\omega}_{s g+p}=A \cos \Omega\left(t-t_{0}\right), \\
& \Delta e_{s g+p}=\Delta e_{s g+p}^{0}-e A \sin \Omega\left(t-t_{0}\right),
\end{aligned}
$$

where $A$ is a constant, and the libration frequency is

$$
\Omega=\frac{m}{\pi M}\left(\frac{a}{\Delta a}\right)^{2} n H\left(q^{2}\right) .
$$

The libration times $2 \pi / \Omega$ are listed in Table I. The existence of libration oscillations shows that the equilibrium solution (28) is dynamically stable (see also Yoder 1983). Note that $\Delta e^{0} / e$ is equal to the ratio of the differential precession frequency to the libration frequency.

\section{b) Viscous Damping and Apsidal Shift}

We consider first the effect of viscous forces on $e_{i}$ and $\widetilde{\omega}_{i}$. From Eqs. (13) and (16)

$$
\begin{aligned}
\left(\frac{d e_{1}}{d t}\right)_{\text {visc }}= & -\left(\frac{d e_{2}}{d t}\right)_{\text {visc }} \\
= & -\frac{4 \pi}{n m}\langle\Sigma\rangle v^{2}\left[\cos \gamma\left(\mathscr{S}_{r r}+2 \mathscr{C}_{r \phi}\right)\right. \\
& \left.-\sin \gamma\left(\mathscr{C}_{r r}-2 \mathscr{S}_{r \phi}\right)\right], \\
\left(\frac{d \widetilde{\omega}_{1}}{d t}\right)_{\text {visc }}= & -\left(\frac{d \widetilde{\omega}_{2}}{d t}\right)_{\text {visc }} \\
= & \frac{4 \pi}{n m e}\langle\Sigma\rangle v^{2}\left[\cos \gamma\left(\mathscr{C}_{r r}-2 \mathscr{S}_{r \phi}\right)\right. \\
& \left.+\sin \gamma\left(\mathscr{S}_{r r}+2 \mathscr{C}_{r \phi}\right)\right] .
\end{aligned}
$$

Thus $(d e / d t)_{\text {visc }}=\frac{1}{2}\left[d\left(e_{1}+e_{2}\right) / d t\right]_{\text {visc }}=0$, and if we work to the same order as in the previous subsection, we have $e=\frac{1}{2}\left(e_{1}+e_{2}\right)=$ constant [but see Eq. (37) below]. Using Eqs. (19) and (25) we may write

$$
\begin{aligned}
& \left(\frac{d \Delta e}{d t}\right)_{\text {visc }}=\lambda_{1} \Delta e+\lambda_{2} e \Delta \widetilde{\omega}, \\
& \left(\frac{d \Delta \widetilde{\omega}}{d t}\right)_{\text {visc }}=\lambda_{1} \Delta \widetilde{\omega}-\frac{\lambda_{2} \Delta e}{e},
\end{aligned}
$$

where

$$
\begin{aligned}
& \lambda_{1}=\frac{2 v^{2}\left(\mathscr{S}_{r r}+2 \mathscr{C}_{r \phi}\right)}{n(\Delta a)^{2} q\left(1-q^{2}\right)^{1 / 2}}, \\
& \lambda_{2}=\frac{2 v^{2}\left(\mathscr{S}_{r r}-2 \mathscr{C}_{r \phi}\right)}{n(\Delta a)^{2} q\left(1-q^{2}\right)^{1 / 2}} .
\end{aligned}
$$

The equilibrium condition $(d \Delta \widetilde{\omega} / d t)_{s g+p+\text { visc }}$ $=(d \Delta e / d t)_{s g+p+\text { visc }}=0$ is obtained from Eqs. (26), (27), and (32):

$$
\begin{aligned}
\Delta \widetilde{\omega}_{s g+p+\text { visc }}^{0}= & \frac{21}{4} J_{2}\left(\frac{R}{a}\right)^{2} n \\
& \times \frac{\Delta a}{a} \frac{\lambda_{1}}{\left(\Omega-\lambda_{2}\right)^{2}+\lambda_{1}^{2}}, \\
\Delta e_{s g+p+\text { visc }}^{0}= & \frac{21}{4} J_{2}\left(\frac{R}{a}\right)^{2} n \frac{\Delta a}{a} e \frac{\Omega-\lambda_{2}}{\left(\Omega-\lambda_{2}\right)^{2}+\lambda_{1}^{2}} .
\end{aligned}
$$

The general solution of Eqs. (26), (27), and (32) for small oscillations is

$$
\begin{aligned}
\Delta \widetilde{\omega}_{s g+p+\text { visc }}= & \Delta \widetilde{\omega}_{s g+p+\text { visc }}^{0} \\
& +A e^{\lambda_{1} t} \cos \left(\Omega-\lambda_{2}\right)\left(t-t_{0}\right) \\
\Delta e_{s g+p+\text { visc }}= & \Delta e_{s g+p+v i s c}^{0} \\
& -e A e^{\lambda_{1} t} \sin \left(\Omega-\lambda_{2}\right)\left(t-t_{0}\right),
\end{aligned}
$$

where $A$ is a constant. Numerical evaluation of $\mathscr{S}_{r r}+2 \mathscr{C}_{r \phi}$ for a wide variety of values of $q$ and $\langle\tau\rangle$ always yields $\mathscr{S}_{r r}+2 \mathscr{C}_{r \phi}<0$; hence $\lambda_{1}<0$. Thus, the librations described by Eq. (35) are damped, and the equilibrium solution (33) is secularly stable. The damping time $\left|\lambda_{1}\right|^{-1}$ is comparable to the spreading time for an unconstrained ring (i.e., the time required for a ring particle to random walk across a distance $|\Delta a|)$.

For simplicity we henceforth restrict ourselves to the case $\lambda_{1}, \lambda_{2} \ll \Omega$, which is likely to obtain in many planetary rings. In this limit $\Delta e_{s g+p+\text { visc }}^{0} \simeq \Delta e_{s g+p}^{0}$, so that the ring mass estimates obtained from Eq. (28) and given in Table I are still valid. In addition, Eqs. (33) and (34) yield

$$
\begin{aligned}
\Delta \widetilde{\omega}_{s g+p+\text { visc }}^{0} & =\frac{\Delta e}{e} \cdot \frac{\lambda_{1}}{\Omega} \\
& =2 \pi\left(\frac{v}{n a}\right)^{2} \frac{M}{m} \frac{\left(\mathscr{S}_{r r}+2 \mathscr{C}_{r \phi}\right)}{q\left(1-q^{2}\right)^{1 / 2} H\left(q^{2}\right)} \frac{\Delta e}{e} .
\end{aligned}
$$

$\Delta \widetilde{\omega}^{0}$ is roughly equal to the square of the libration period divided by the product of the differential precession time and the spreading time.

We have evaluated Eqs. (33a) and (36) using the approximation $q=a \Delta e / \Delta a$, which is valid for $\left|\lambda_{1}\right|$, $\left|\lambda_{2}\right| \ll \Omega$. We have taken $m, e$, and $\Delta e$ from Table I and assumed $\langle\tau\rangle=1.0$ for all rings (our results are not very sensitive to $\langle\tau\rangle$; reducing $\langle\tau\rangle$ to 0.5 increases $\left|\Delta \widetilde{\omega}^{0}\right|$ by a factor of 1.4). The resulting damping times and apsidal shifts are given in Table I for a representative value $v=0.1 \mathrm{~cm} / \mathrm{s}$. Recall that the total shift between the inner and outer edges of the ring is $2 \Delta \widetilde{\omega}^{0}$, assuming that $\widetilde{\omega}$ is a linear function of semimajor axis. The values of $\Delta \widetilde{\omega}^{0}$ are negative, so that the inner apse precedes the outer. Although the predicted apsidal shifts are rather small, the difference between the azimuth of maximum ring width and the azimuth of maximum distance from the planet is $\Delta \phi=\tan ^{-1}(e \Delta \widetilde{\omega} / \Delta e)$, which can be much 
larger. (Note that $\Delta \phi=-\gamma$ according to Eq. 24. Values of $\gamma$ are listed in Table I.) It may be possible to measure $\Delta \phi$ directly in the $\alpha$ and $\beta$ rings of Uranus (Nicholson 1982).

The assumption that $\left|\lambda_{1}\right|,\left|\lambda_{2}\right| \ll \Omega$, on which Table I is based, is only self-consistent if $|\tan \gamma| \ll 1$. This assumption is only marginally valid for the $\alpha$ and $\beta$ rings if $v=0.1 \mathrm{~cm} / \mathrm{s}$. For larger velocity dispersions the analysis must be based on Eqs. (34) instead of Eq. (36). More sophisticated data analysis is also required, since the values of $2 \Delta \mathrm{e}$ and $2 \Delta a$ in Table I are based on the assumption of apse alignment, which also is valid only for $|\tan \gamma| \ll 1$.

\section{c) Eccentricity Damping}

In the previous section we showed that viscous forces damp librations in the two-streamline model. However, the mean eccentricity $e$ was conserved. To analyze damping of the mean eccentricity requires a slightly more careful treatment.

In writing Eqs. (26) from Eqs. (5) and (8), we kept only the largest nonzero terms in $\Delta e$ and $\Delta \widetilde{\omega}$. If we keep terms smaller by $\Delta e / e=0\left(\epsilon_{1} / \epsilon_{2}\right)$, then Eq. (5a) yields

$$
\frac{d e}{d t}=\frac{1}{2} \frac{d\left(e_{1}+e_{2}\right)}{d t}=\frac{m}{4 \pi M}\left(\frac{a}{\Delta a}\right)^{2} n H\left(q^{2}\right) \Delta e \Delta \widetilde{\omega} .
$$

Since $\Delta \widetilde{\omega}<0$, the mean eccentricity is damped. The damping time is just $4(e / \Delta e)^{2}$ times the damping time for the libration. Using Eqs. (28), (30), and (36), in the limit $\left|\lambda_{1}\right|,\left|\lambda_{2}\right| \ll \Omega$, the damping time $t_{\mathrm{damp}}=\left|e^{-1} d e / d t\right|^{-1}$ may be written

$$
\begin{aligned}
t_{\text {damp }}^{-1} & =-\frac{\lambda_{1}}{4}\left(\frac{\Delta e}{e}\right)^{2}, \\
& =\frac{21}{16} J_{2}\left(\frac{R}{a}\right)^{2} \frac{n}{a}|\Delta \widetilde{\omega} \Delta a| .
\end{aligned}
$$

In equilibrium the eccentricity damping rate described by Eq. (37) is presumably balanced by eccentricity pumping by the shepherd satellites (Eq. 21).

These results can be interpreted using a simple physical argument. The rate of energy dissipation per unit mass in a viscous fluid is of order $\dot{E} \sim v S^{2}$, where $v$ is the kinematic viscosity and $S$ is the shear. The excess shear caused by the eccentricity gradient is $\sim a n \Delta e / \Delta a$, leading to an enhancement of the energy dissipation rate by $\Delta \dot{E} \sim v(a n \Delta e / \Delta a)^{2}$. The excess energy contained in an eccentric ring is $\sim(n a e)^{2}$ per unit mass, which can be dissipated by $\Delta \dot{E}$ in a time $t_{\text {damp }}$ given by $t_{\text {damp }}^{-1} \sim v(\Delta e)$ $e \Delta a)^{2}$. Since $\Delta a^{2} / v$, the spreading time for an unconstrained ring, is roughly equal to $\left|\lambda_{1}\right|^{-1}$, we have $t_{\text {damp }}^{-1} \sim\left|\lambda_{1}\right|(\Delta e / e)^{2}$, recovering Eq. (38a).

\section{IV. $N$-STREAMLINE MODELS}

The two-streamline model of the previous section provides a useful qualitative picture of the evolution of eccentric rings. However, quantitative results require numerical $N$-streamline models based on the equations of Sec. II, which will be described in a subsequent paper.

In this section we describe a general proof of the relation between apsidal shift and eccentricity damping, valid for these models.

We divide the ring into $N$ streamlines, each of mass $m_{i}=m / N$. We introduce the complex eccentricity

$$
\zeta=e \exp (I \widetilde{\omega})
$$

where $I^{2}=-1$, and write $\bar{\zeta}=N^{-1} \Sigma_{i} \xi_{i}$. If we neglect terms of $O(e)$ in Gauss's equations (12), the rate of change of $\zeta$ is

$$
\frac{d \zeta}{d t}=\frac{\exp (I \phi)}{n a}(2 T-I S)
$$

Since the viscous forces between adjacent streamlines are equal and opposite at every azimuth $\phi$, and the masses of the streamlines are equal, $\left[d\left(\xi_{i} \pm \zeta_{i+1}\right) / d t\right]_{\text {visc }}=\left[d\left(\zeta_{i}+\zeta_{i-1}\right) / d t\right]_{\text {visc }}=0$.

Thus $\bar{\xi}$ is conserved under the influence of viscous forces. [A more careful analysis based on the exact Gauss equations shows that $(d \bar{\zeta} / d t)_{\text {visc }}=O\left(v^{2} \Delta e / n a^{2}\right)$, a rate which is negligible on the time scales of interest in this paper. A similar result was obtained by Goldreich and Tremaine 1981.]

The rate of change of $\xi_{i}$ due to the gravitational force from streamline $j$ is given by Eq. (5):

$$
\left(\frac{d \zeta_{i}}{d t}\right)_{s g}=I C_{i j} H\left(q_{i j}^{2}\right)\left(\zeta_{i}-\zeta_{j}\right)
$$

Since $C_{i j}$ and $q_{i j}$ are symmetric in $i$ and $j$, $\left[d\left(\zeta_{i}+\zeta_{j}\right) / d t\right]_{s g}=0$. Thus, $\bar{\zeta}$ is also conserved under gravitational forces within the ring. This result depends on the condition that the ring is narrow.

The rate of change of $\xi_{i}$ due to the planetary quadrupole moment is

$$
\left(\frac{d \zeta_{i}}{d t}\right)_{p}=I \zeta_{i}\left(\dot{\tilde{\omega}}_{i}\right)_{p}
$$

where $\left(\dot{\tilde{\omega}}_{i}\right)_{p}$ is given by Eq. (8a). Thus the total rate of change of $\xi$ is solely due to the differential precession, and

$$
\frac{d \bar{\zeta}}{d t}=\left(\frac{d \bar{\zeta}}{d t}\right)_{p}=\frac{I}{N} \sum_{i=1}^{N} \zeta_{i}\left(\dot{\tilde{\omega}}_{i}\right)_{p}
$$

The mean eccentricity of the ring $e$ is given by $e^{2}=|\bar{\zeta}|^{2}$. Hence the eccentricity damping rate is given by

$$
\begin{aligned}
\frac{d e^{2}}{d t} & =\bar{\zeta} \frac{d \bar{\zeta}^{*}}{d t}+\bar{\zeta} * \frac{d \bar{\zeta}}{d t} \\
& =\frac{I}{N^{2}} \sum_{i, j=1}^{N} e_{i} e_{j} \exp \left[I\left(\widetilde{\omega}_{j}-\widetilde{\omega}_{i}\right)\right]\left[\left(\dot{\widetilde{\omega}}_{j}\right)_{p}-\left(\left(\dot{\tilde{\omega}}_{i}\right)_{p}\right]\right.
\end{aligned}
$$

If the variation in $e$ and $\widetilde{\omega}$ across the ring is small, we may set $e_{i} \simeq e_{j} \simeq e$ and $\exp \left[I\left(\widetilde{\omega}_{j}-\widetilde{\omega}_{i}\right)\right]$ $\simeq 1+I\left(\widetilde{\omega}_{j}-\widetilde{\omega}_{i}\right)$, so that 


$$
\frac{1}{e} \frac{d e}{d t}=-\frac{1}{2 N^{2}} \sum_{i, j=1}^{N}\left(\widetilde{\omega}_{j}-\widetilde{\omega}_{i}\right)\left[\left(\dot{\tilde{\omega}}_{j}\right)_{p}-\left(\dot{\tilde{\omega}}_{i}\right)_{p}\right]
$$

Using Eq. (8) we have

$$
\frac{1}{e} \frac{d e}{d t}=+\frac{21}{8} J_{2}\left(\frac{R}{a}\right)^{2} \frac{n}{a N^{2}} \sum_{i, j=1}^{N} \Delta \widetilde{\omega}_{j i} \Delta a_{j i} .
$$

This result directly relates the eccentricity damping to the apsidal shift. For $N=2$ we recover Eq. (38). If we assume that $\widetilde{\omega}$ is a linear function of $a$, that the total ring width and apse shift are $2 \Delta a$ and $2 \Delta \widetilde{\omega}$, and that the ring has uniform surface density, then

$$
\frac{1}{t_{\mathrm{damp}}}=\left|\frac{1}{e} \frac{d e}{d t}\right|=\frac{7}{4} J_{2}\left(\frac{R}{a}\right)^{2} \frac{n}{a}|\Delta \tilde{\omega} \Delta a| .
$$

The difference between Eqs. (38) and (47) is a crude measure of the uncertainty in our result, which can be eliminated by numerical calculations.

\section{DISCUSSION}

The simple model of Sec. III leads to the following qualitative picture of the evolution of eccentric rings: The mean eccentricity of a nearly circular ring will grow if the strongest corotation resonances with the shepherd satellites are saturated or if gaps form at the strongest Lindblad resonances (Eq. 21). As the mean eccentricity grows, viscosity damps the librations of the eccentricity and apse differences, so that $\Delta e$ and $\Delta \widetilde{\omega}$ settle into the equilibrium values required for uniform precession (Eq. 34). The mean eccentricity continues to grow until excitation by the shepherds is balanced by viscous damping (Eq. 37).

This scenario is subject to several comparisons with observations:

(1) If uniform precession is maintained by self-gravity then $\Delta e / \Delta a>0$ (cf. Eq. 28 and Paper I). This relation is satisfied in all elliptical rings with measured $\Delta e$ and $\Delta a$.

(2) The equilibrium Eq. (28) predicts masses and surface densities for the eccentric Saturn ringlets. These surface densities are consistent with independent estimates based on the wavelengths of density waves (Cuzzi et al. 1981; Holberg et al. 1982) and the scattering of the Voyager 1 radio signal (Tyler 1982) at other places in the rings.

(3) The predicted apsidal shifts $\Delta \widetilde{\omega}^{0}$ (Eq. 36) may be detectable in the $\alpha$ and $\beta$ rings of Uranus.
(4) Combining Eqs. (28), (36), and (37), the eccentricity damping rate becomes

$$
\begin{aligned}
t_{\text {damp }}^{-1} & =\left|\frac{1}{e} \frac{d e}{d t}\right| \\
& =\frac{n}{2}\left[\frac{21 \pi}{4} \frac{M}{m} J_{2}\left(\frac{R}{a}\right)^{2}\right]^{2} \\
& \times\left\{\left(\frac{\Delta a}{a}\right)^{4}\left(\frac{v}{n a}\right)^{2} h(q,\langle\tau\rangle)\right\},
\end{aligned}
$$

where

$$
h(q,\langle\tau\rangle)=-\frac{\left(\mathscr{S}_{r r}+2 \mathscr{C}_{r \phi}\right)}{H\left(q^{2}\right)^{2} q\left(1-q^{2}\right)^{1 / 2}} .
$$

The eccentricity pumping by the shepherd satellites (Eq. 21 ) is not directly dependent on $q$, nor are the factors in square brackets in Eq. (48). For $|q|<1, \mathscr{S}_{r r}+2 \mathscr{C}_{r \phi}$ is proportional to $q$, so that $h(q,\langle\tau\rangle)$ is independent of $q$. The equilibrium coefficient of restitution $\epsilon$ and velocity dispersion $v$ are also independent of $q$ in this regime. Thus the damping time $t_{\text {damp. }}$ (Eq. 48) is independent of $q$ for $|q|<1$. As $|q|$ approaches unity, $h(q ;\langle\tau\rangle)$ is a slowly decreasing function of $|q|$, while the velocity dispersion increases with $|q|$ (Borderies et al. 1983c). Without improved data on the $\epsilon-v$ relation for the ring material (and more detailed models of the ring dynamics) it is difficult to predict the equilibrium value of $|q|$. However, the arguments above suggest that the equilibrium values of $|q|$ should be of order unity, as is observed for the rings in Table I (assuming $q \simeq a \Delta e / \Delta a$, which is valid if $\left|\lambda_{1}\right|$, $\left|\lambda_{2}\right|<\Omega$ and if $q$ is constant across the ring*).

We thank J. Elliot, P. Nicholson, C. Porco, and C. Yoder for discussions. N. B. acknowledges a NSF U.S.France exchange postdoctoral fellowship and a grant from ATP Planétologie 1982. P. G. acknowledges support from NSF Grant AST 80-20005 and NASA Grant NGL-05-002-003. S. T. acknowledges support from NSF Grant AST-8210463 and NASA Grants NSG7643 and NGL-22009-638.

*In fact $q$ is probably not constant, and the maximum value of $q$ in each ring is likely to be substantially larger than the values determined from the ring boundaries. For example, in model (d) of Paper I the maximum value of $q$ in the $\epsilon$ ring was 0.8 , compared to 0.65 determined from the ring boundaries.

\section{REFERENCES}

Borderies, N., Goldreich, P., and Tremaine, S. (1983a). Astron. J. 88, 226.

Borderies, N., Goldreich, P., and Tremaine, S. (1983b). Icarus, to be published.

Borderies, N., Goldreich, P., and Tremaine, S. (1983c). Icarus, to be published.

Cuzzi, J. N., Lissauer, J. J., and Shu, F. H. (1981). Nature 292, 703.

Esposito, L. W., Borderies, N., Goldreich, P., Cuzzi, J. N., Holdberg,
J. B., Lane, A. L., Pomphrey, R. B., Terrile, R. J., Lissauer, J. J. Marouf, E. A., and Tyler, G. L. (1983). Science, to be published. French, R. G., Elliot, J. L., and Allen, D. A. (1982). Nature 298, 827. Goldreich, P., and Tremaine, S. (1978). Icarus 34, 227. Goldreich, P., and Tremaine, S. (1979a). Nature 277, 97. Goldreich, P., and Tremaine, S. (1979b). Astron. J. 84, 1638 (Paper I). Goldreich, P., and Tremaine, S. (1981). Astrophys. J. 243, 1062. Holberg, J. B., Forrester, W. T., and Lissauer, J. J. (1982). Nature 297, 
115.

Nicholson, P. D. (1982). Private communication.

Nicholson, P. D., Matthews, K., and Goldreich, P. (1982). Astron. J. 87, 433.

I-1 Porco, C. (1983). Ph.D. thesis, California Institute of Technology.

Porco, C., Borderies, N., Danielson, G. E., Goldreich, P., Holberg, J.
B., Lane, A. L., and Nicholson, P. D. (1983). In I.A.U. Colloquium No. 75, Planetary Rings, edited by A. Brahic and R. A. Greenberg. Tyler, L. (1982). Private communication.

Yoder, C. F. (1983). In I.A.U. Colloquium No. 75, Planetary Rings, edited by A. Brahic and R. A. Greenberg. 